\title{
Studies of QCD jet production with the CMS detector in pp Collisions at $\sqrt{s}=7 \mathrm{TeV}$
}

\author{
Mikko Voutilainen* \\ CERN, for the CMS collaboration \\ E-mail: mikko.voutilainen@cern.ch
}

\begin{abstract}
We report on an extensive list of analyses that test QCD predictions for jet production in pp collisions at $\sqrt{s}=7 \mathrm{TeV}$, recorded by the CMS experiment. The list includes a measurement of the inclusive jet spectra, obtained with different jet reconstruction methods, the ratio of the inclusive three-jet over two-jet cross sections as a function of the total jet transverse momentum $H_{T}$, hadronic event shapes as determined from jet momenta, azimuthal decorrelations between the two leading jets, and dijet angular distributions. Finally, we also present a study of the jet transverse structure, the charged hadrons multiplicity in jets and the longitudinal and transverse momentum distribution of charged hadrons relative to the jet axis. Many of these analyses are based on ratio quantities, where important experimental systematic uncertainties and most notably the luminosity uncertainty cancel.
\end{abstract}

35th International Conference of High Energy Physics - ICHEP2010,

July 22-28, 2010

Paris France

* Speaker. 


\section{Introduction}

We report on an extensive list of QCD measurements [1] performed on the initial p-p data collected by the CMS experiment. The first $\sqrt{s}=7 \mathrm{TeV}$ collisions at CERN took place on May $30^{\text {th }}$ 2010, and approximately $100 \mathrm{nb}^{-1}$ were collected by mid-July. With an exponentially rising luminosity, most of this data was collected within the two weeks leading to the conference. With wellworking detector, simulation, reconstruction and data quality management, this data was promptly analyzed into good quality physics results. Starting from inclusive jet cross section, more specific event topologies focus on different aspects of the QCD theory. Many of these measurements are ratio quantities, which reduces sensitivity to experimental uncertainties from JEC and luminosity.

\section{Methods}

The jet measurements at CMS are performed with three types of jets: calorimeter (CALO) jets [2], jet-plus-track (JPT) jets [3] and particle flow (PF) jets [4]. All jet types are clustered with the anti- $k_{T}$ algorithm [5] with radius of 0.5 and 0.7. The calorimeter jets use as input calorimeter towers reconstructed from hadronic calorimeter (HCAL) cells and a $5 \times 5$ matrix of electromagnetic calorimeter (ECAL) crystals. The JPT algorithm starts from CALO jets and performs corrections for tracks based on the expected calorimeter response to improve response and resolution. The PF algorithm, very specific to CMS, uses a heuristic combination of HCAL cells, ECAL crystals and matched tracks to optimally reconstruct individual particles before jet clustering. The baseline jet energy corrections for each jet reconstruction method are derived using MC simulation, with residual corrections for rapidity dependence derived using dijet $p_{T}$ balance. The a-priori JEC uncertainties, $10 \%$ for CALO jets and 5\% for tracking-based jets [2], have been validated using photon+jet data. The jets are collected using a combination of Minimum Bias and single jet triggers to cover $p_{T}$ range of $18 \mathrm{GeV}$ to about $600 \mathrm{GeV}$ with $60 \mathrm{nb}^{-1}$ of data. The uncertainty on the integrated luminosity is $11 \%$, with largest contribution from the LHC beam current measurements.

\section{Measurements}

For the inclusive jet cross section measurement jets are binned in transverse momentum $p_{T}$ and rapidity $y$. The measured spectra are unfolded with the ansatz method, with jet energy resolutions (JER) taken from Pythia MC. The MC resolutions have been validated from data to within $10 \%$ uncertainty [2]. The unfolding corrections range from $20 \%$ at low $p_{T}$ to about $5 \%$ at high $p_{T}$ in the barrel at $|y|<1.0$. The results for different jet reconstruction types are compared to next-toleading order (NLO) theory predictions in Fig. 1. Good agreement is found within the experimental uncertainty of about $20-30 \%$, and theoretical uncertainty of 5-10\% from scale and parton distribution function uncertainty and up to $50 \%$ at low $p_{T}$ from non-perturbative corrections. The latter is estimated as half-difference between Pythia [6] and Herwig [7] predictions.

The 3-jet and 2-jet cross section ratio measurement were done using jets with $p_{T}>50 \mathrm{GeV}$ within the central calorimetry at $|y|<2.5$. The measurement was performed as a differential with respect to the jet scalar sum $E_{T}$ denoted as $H_{T}$. The high $p_{T}$ plateau region of this measurement is sensitive to the strong coupling constant $\alpha_{S}$, while the lower $p_{T}$ region is sensitive to MC modeling. Good agreement is found with Pythia 6 and MadGraph [8] predictions within uncertainties. 

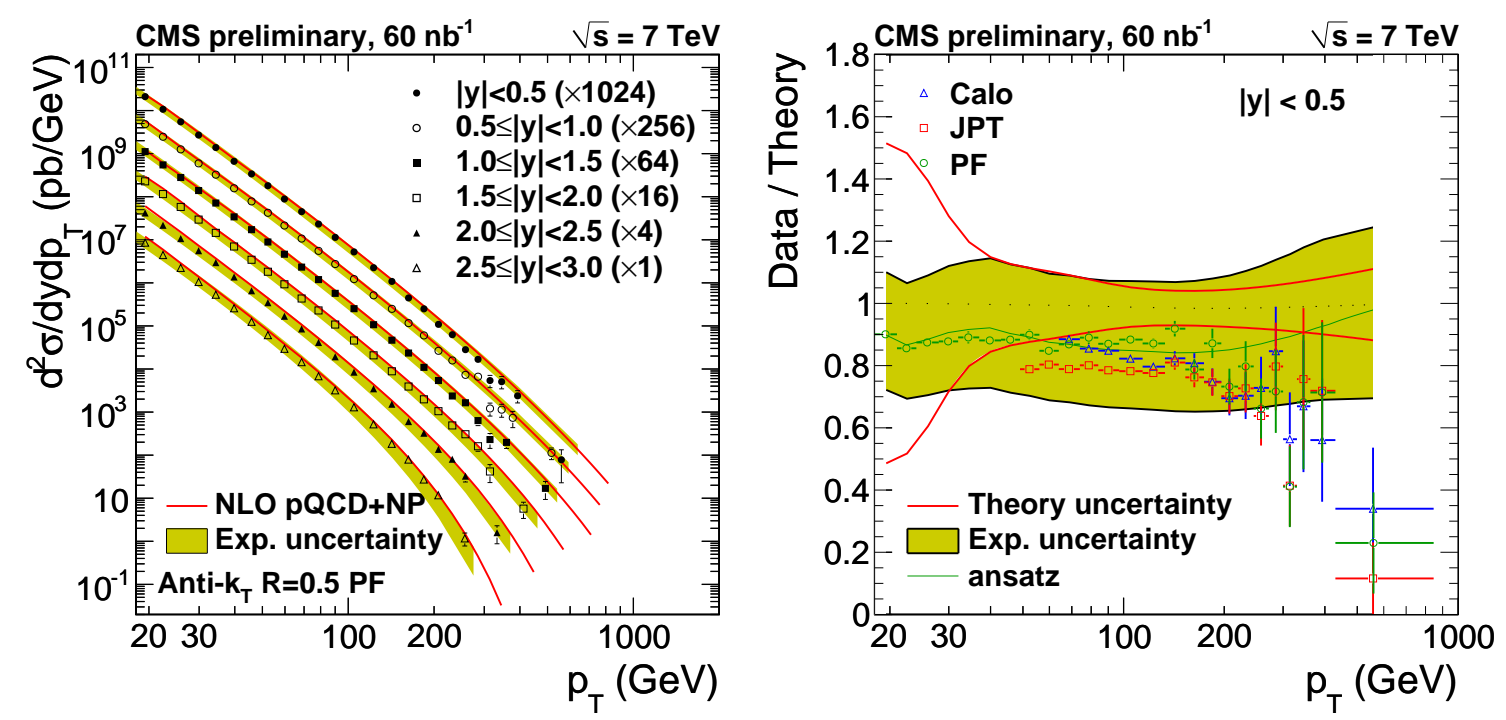

Figure 1: Measurement of inclusive jet cross section (left), and ratio of data and NLO theory prediction for different methods of jet reconstruction (right).

The hadronic events shapes provide geometric information about the energy flow in hadronic events. This gives essential feed-back for tuning parton shower and non-perturbative components of MC event generators. The event shape variables show good agreement between different jet reconstruction types. As shown in Fig. 2, Pythia 6 and Herwig++ agree with data within uncertainties; Alpgen [9] and MadGraph overestimate the fraction of back-to-back dijets, while Pythia 8 underestimates it. The results are similar at all probed jet $p_{T}$ ranges at $|\eta|<1.3$.

The dijet azimuthal decorrelation measures the angle $\Delta \phi_{\text {dijet }}$ between the two leading jets in the transverse plane. This quantity is very sensitive to initial state radiation $\left(k_{I S R}\right)$, as shown in Fig. 2, but shows little sensitivity to final state radiation and to experimental systematics from JEC or luminosity. Comparisons between data and different models show good agreement with Pythia default tune $\left(k_{I S R}=2.5, k_{F S R}=4.0\right)$ and Herwig++, but less agreement with MadGraph at low $p_{T}$.

The dijet angular distributions are a sensitive probe of new physics, e.g. contact interactions, which are expected to produce jets isotropically $\left(y_{1} \approx y_{2}\right)$ and peak at low $\chi_{\text {dijet }}=\exp \left(\left|y_{1}-y_{2}\right|\right)$. The QCD jet production is mostly t-channel and hence flat in $\chi_{\text {dijet }}$. The measurement has sensitivity up to contact interaction scale of $\Lambda=3 \mathrm{TeV}$ with few $\mathrm{pb}^{-1}$, compared to Tevatron limits of $\Lambda>2.8-3 \mathrm{TeV}$. For the current data set of $16 \mathrm{nb}^{-1}$ good agreement is found between data and NLO theory prediction within experimental and theoretical uncertainties.

The jet transverse shapes probe transition between hard perturbative QCD and soft gluon radiation. This transition is described by phenomenological models motivated by QCD and tuned at $e^{+} e^{-}$colliders. At hadron colliders underlying event is an important ingredient. The models have been tuned at the Tevatron for $\sqrt{s}=2 \mathrm{TeV}$, but the extrapolation to higher LHC energies is uncertain. The results from integrated jet shapes, charged particle multiplicity and jet width are compared to Pythia and Herwig++ predictions and data is found to be consistent with both at $p_{T}>50 \mathrm{GeV}$ within experimental uncertainties. At lower $p_{T}$ Pythia predicts slightly too broad jets, Herwig slightly too narrow. The integrated jet shapes are sensitive to the underlying event, but 

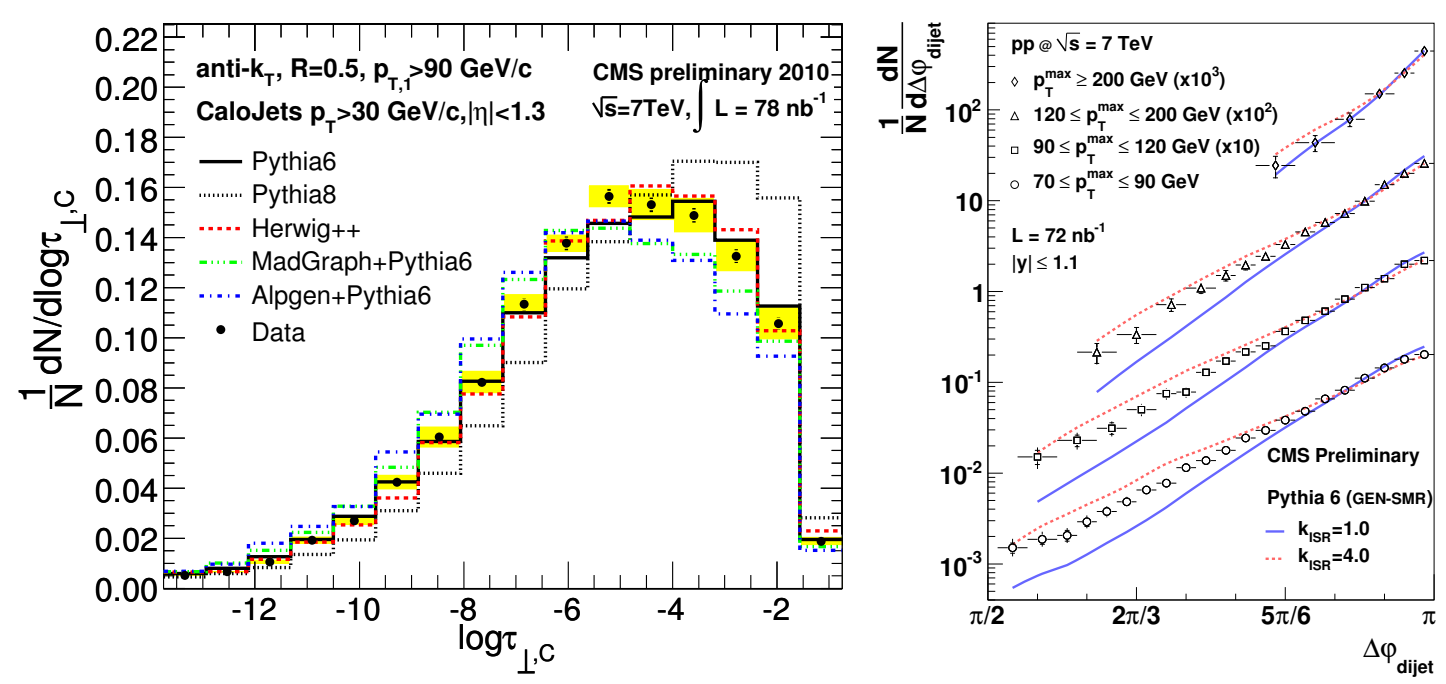

Figure 2: Left: hadronic event shapes. Small values of $\log \left(\tau_{\perp, C}\right)$ correspond to dijet topologies, higher values to multijets. Right: dijet azimuthal decorrelations.

not yet precise enough to differentiate between theoretical models.

\section{Conclusions}

We reported on an extensive list of QCD studies, finding that both the detector and the detector simulation work well. Three different jet reconstruction methods based on different combinations of calorimetry and tracking were commissioned and successfully used in analysis. The most widely used MC tunes at CMS, Pythia D6T and Herwig++, were found to be in general good agreement across all measurements. They were in particular favored by measurements of the hadronic event shapes and dijet azimuthal decorrelations, while Pythia 8, MadGraph and Alpgen showed some differences to data. Agreement was also found between data and next-to-leading order theory for the inclusive jet production and dijet angular distributions.

\section{References}

[1] CMS Collaboration: CMS PAS QCD-10-011, QCD-10-012, QCD-10-013, QCD-10-014, QCD-10-015 (2010).

[2] CMS Collaboration, CMS PAS JME-10-003 (2010).

[3] CMS Collaboration, CMS PAS JME-09-002 (2009).

[4] CMS Collaboration: CMS PAS PFT-09-001 (2009), PFT-10-002 (2010).

[5] M. Cacciari, G. P. Salam, and G. Soyez, JHEP 0804:063 (2008).

[6] T. Sjostrand, S. Mrenna, and P. Skands, JHEP 05:026 (2007).

[7] M. Bahr et al., Eur. Phys. J. C58 (2008) 639707.

[8] J. Alwall et al., JHEP 09:028 (2007).

[9] M. L. Mangano, M. Moretti, F. Piccinini et al., JHEP 07:001 (2003). 